\title{
A VERSATILE METHOD OF BEAMLINE CONSTRUCTION*
}

\author{
Kevin J. Beczek ${ }^{\dagger}$, John W. Lewellen \\ Argonne National Laboratory, Advanced Photon Source, \\ 9700 S. Cass Avenue, Argonne, IL 60439 USA
}

\section{Abstract}

At the Advanced Photon Source (APS), a method for beamline construction has been developed that allows the researcher to reconfigure the beamline quickly and easily. This technique has been extensively incorporated into the APS injector test stand [1]. Rather than use a statically designed system, it was decided at the beginning of the project that an approach that emphasized versatility would provide maximum utility. A concise review of similar previous approaches at APS will be explored, followed by a presentation of this method and other possible variations, with a bias toward fabrication, assembly, and operator considerations.

\section{INTRODUCTION}

If necessity is truly the mother of invention, then we physicists and engineers must have many needs. Four such needs manifested themselves during the planning stages of the APS injector test stand [1] in the fall of 2000. Two of these requirements came to mind immediately. First, the means of supporting and aligning the components of the test stand had to use standardized, "off the shelf" parts as much as possible. Second, the repeatability of the components had to be within 25 microns transverse to the beam. Initially this stand and the room enveloping it was envisioned to be used as an accelerator testing center, focusing on new injector designs. Based on past experience, however, we decided to provide for ease of reconfiguration and addition. Third, we wanted a construction method not only for experiments that we knew we wanted to perform, but more importantly, for experiments that we could not foresee in the present. Finally, the technique had to lend itself to being assembled and reconfigured in a variety of ways quickly and easily. Above all, the method had to allow complete interchangeability of all major beamline components, especially our newly manufactured thermionic-cathode $\mathrm{rf}$ electron guns, [2] with their counterparts on each of the two existing gun-to-linac (GTL) transport lines $[3,4]$.

\footnotetext{
*Work supported by the U.S. Department of Energy, Office of Basic Energy Sciences, under Contract No. W-31-109-ENG-38.

†beczek@aps.anl.gov
}

\section{HISTORICAL BACKGROUND}

While precision positioning mechanisms have been used quite often and successfully at APS, the use of linear bearings as structural elements in accelerator design is a relatively recent innovation. We hoped that experience with modular component positioning mechanisms designed for the LEUTL Project $[5,6]$ might serve as a viable solution. At first glance, this arrangement appeared to fill most of the outlined criteria. However, the cross section of the rails used for the Low-Energy Undulator Test Line (LEUTL) is asymmetric; quite different from the GTL supports. This feature would not allow the GTL components to be interchanged with those on the test stand. Much of the final concept stems from the approach used to support and align the APS injectors. Figure 1 shows the existing GTL support structure. Notice the twin Thomson [7] linear guide rails supporting all of the magnets, diagnostics, and injector.

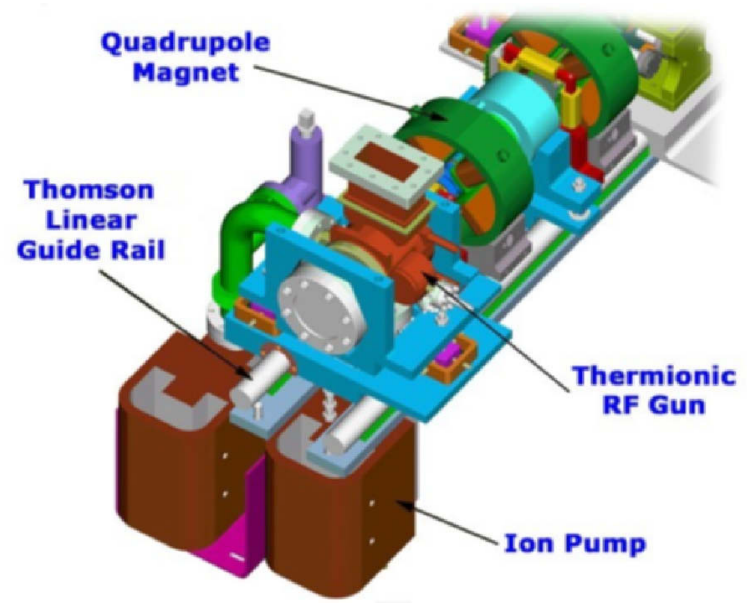

Figure 1: Existing gun-to-linac (GTL) transport line support.

\section{DETAILED DESCRIPTION}

\subsection{Carriage and Rail System Similitude}

Let us examine the mechanical similarity between the GTL supports and the test stand supports. Each accelerator component is rigidly fastened to a carriage that sits on top of the two rails at equivalent height (Y-direction), as shown in Figure 2. Notice that only one rail is captured to constrain movement in the X-direction; placing constraints on both 
rails would result in a redundancy of location, preventing the carriage from seating precisely. This is sometimes referred to in manufacturer's literature as a "master-slave" relationship. The master is the more constrained of the pair. The $\mathrm{Z}$ position is, at least in this case, placed manually, although it is indeed possible to automate this system using, for instance, stepper motors with an appropriate feedback loop to control the carriage. Once the desired $\mathrm{Z}$ position is reached, the carriage is then bolted down tightly against the top surface of the two rails from the underside of the assembly. The shortcomings of this previous design were addressed, and expandability and ease of fabrication then became prime concerns.

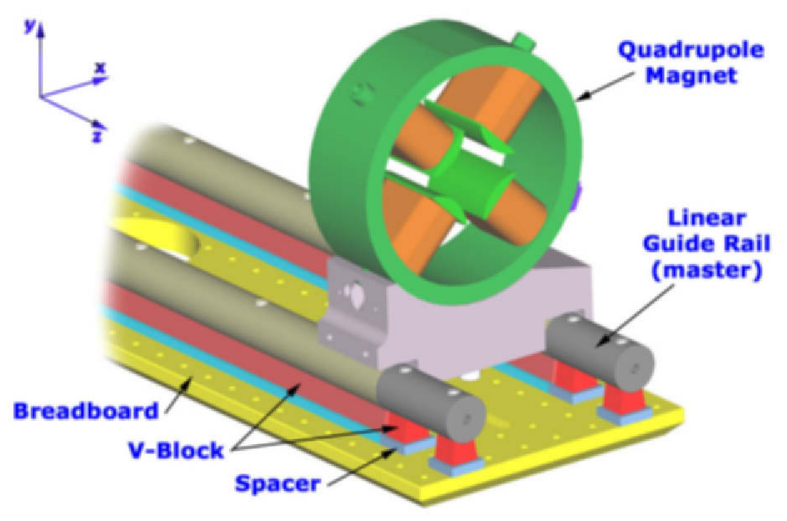

Figure 2: Quadrupole magnet on carriage.

\subsection{Modular Guide Rail and V-Block}

It is from this point that the supports developed for the test stand depart from the GTL supports. There are five distinct differences as illustrated in Figure 3. First, the product is easily connected end to end using a standard size dowel pin or spring pin. The desired length, therefore, is not limited because additional rails may be "pinned" on. Those who purchase the Thomson product will find this may only be accomplished through costly custom fabrication. Second, all of the v-block supports beneath the guide rails are the same length. This may sound simplistic, but consider that in the original GTL transport line design each v-block cradled the guide rail for its entire length and had to be cut or purchased for that particular length of rail. Slicing them into a compact, uniform size and spacing them at a specific distance apart makes each v-block completely independent of the rail length. Bear in mind that because this design lends itself to mass production techniques, the cost per unit to fabricate them is reduced substantially. Third, because the loading conditions are static, or very low cyclic at most, it becomes unnecessary to case-harden the guide rail as with the Thomson rail. This allowed us to use a much less expensive, yet highly precise, class of material usually reserved for mechanical drive shafts. When we measured the assembled parts, the height and diameter fell within a 12micron silhouette, validating our choice. Fourth, the guide rails of the GTL line were assembled from the bottom of the support. This was altogether eliminated in the test stand and replaced with guide rails fastened from the top of the support. This greatly improves access to modify the system and is well suited to the multiple layered breadboard idea described below. Finally, because the components are part of a "family" of various lengths, it is possible for the researcher to interchange mating rails and configure the beamline in any length desired.

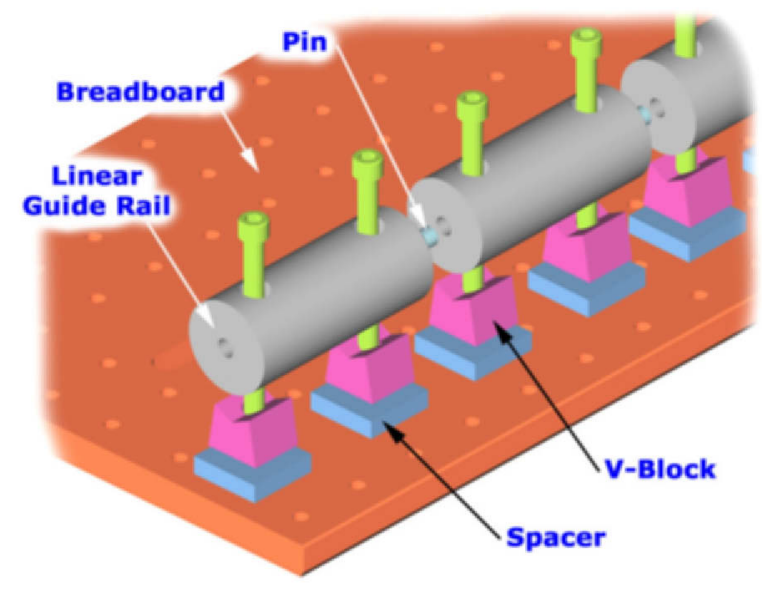

Figure 3: Exploded view of assembly.

\subsection{Multiple Layered Breadboard}

The guide rails and v-blocks have been designed for installation onto a standard hole pattern breadboard $(1 " \times 1$ " centers with 1/4-20 UNC thread). Breadboards have been used for many years in the optics and electronics industries. In our case, we found that by stacking breadboards on a $1 \mathrm{~m}$ $\times 3 \mathrm{~m}$ vibration damping optics table, in effect on top of each other, we could further enhance our ability to reconfigure the beamline supports while using standardized optics parts such as table clamps. Thus, the optics table becomes a sort of "motherboard" with the children fastened to the mother from above. Each breadboard and table can be fastened to each other in the parallel and perpendicular planes, providing very large working surfaces.

\section{SUMMARY}

A versatile method of beamline construction has been presented. This system has been used extensively in designing and building the APS injector test stand, shown entirely in Figure 4. The illustration shows a Pro/Engineer [8] assembly model of the test stand with a $1.83-\mathrm{m}$-tall mannequin for scale. Notice that the breadboards and modular guide rails supporting the beamline components can be positioned diagonally on the optics table. As of the time of writing, U.S. patent protection is being sought. 


\section{ACKNOWLEDGMENTS}

The author would like to thank George Goeppner, Frank DePaola and the many technicians and skilled tradesmen, especially Michelle Givens, Steve Hanuska, Cliff Pitts, Wayne Michalek, Aaron Lopez, Val Svirtun, Mark Martens, Don Sandberg, Ralph Kuechler, John Graham, and Bill Jansma. Their hard work and dedication to this effort is greatly appreciated. Also, thanks to Catherine Eyberger and Eileen Johnson for their patience with me in the editing of this paper.

\section{REFERENCES}

[1] J. Lewellen, K. Beczek, W. Berg, G. Goeppner, A.Grelick, J. Maclean, A. Nassiri, "The Advanced Photon Source Injector Test Stand," these proceedings.
[2] K. Beczek and J. Lewellen, "A Rationalized Approach to Thermionic RF Gun Design," these proceedings.

[3] J. Lewellen et al., "Operation of the APS RF Gun," proc. 1998 Linear Accel. Conf., Chicago, p. 863, 1998.

[4] J. Lewellen, et al., "A Hot Spare Injector for the APS Linac," proc. 1999 IEEE Part. Accel. Conf. New York, p. $1979,1999$.

[5] S. Milton et al., "The Advanced Photon Source Low Energy Undulator Test Line,” Proc. 1997 IEEE Part. Accel. Conf. Vancouver, p. 877, 1997.

[6] K. Beczek et al., "Modular Component Positioning Along the Beamline Axis," Proc. 1999 IEEE Part. Accel. Conf. New York, p. 1378, 1999.

[7] Thomson Industries Inc., 2 Channel Drive, Port Washington, NY 11050 USA.

[8] Pro/Engineer is a registered trademark of Parametric Technology Inc., Waltham, MA USA.

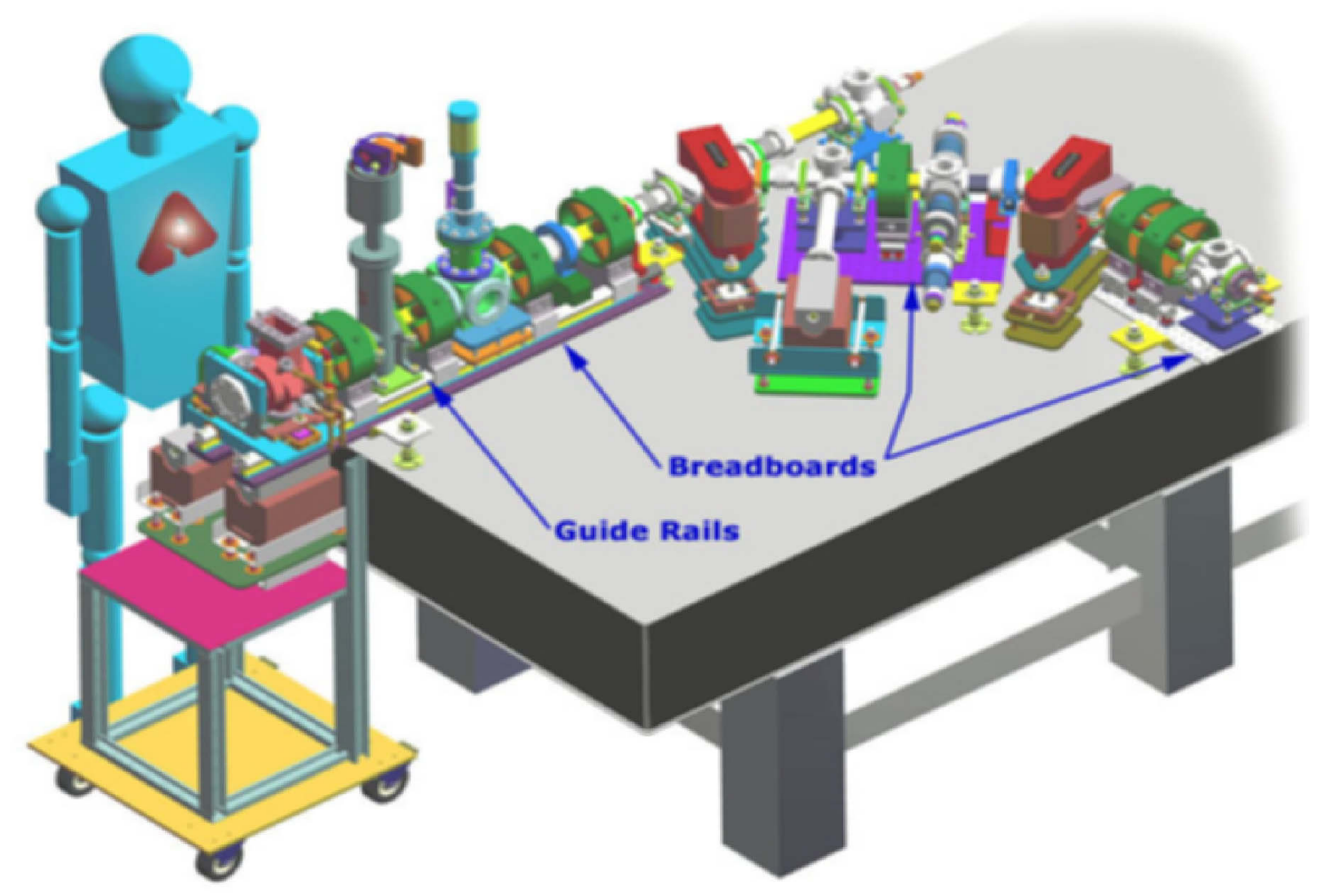

Figure 4: Modular guide rails and v-block supports and multiple layered breadboards as used on APS injector test stand. 